\title{
Experiencia didáctica empírica sobre la clase invertida en el área de Educación Física \\ Empirical didactic experience about flipped classroom on Physical Education area
}

Nerea Felgueras Custodio, M anuel Delgado Pintor

U niversidad Rey Juan Carlos (España)

\begin{abstract}
Resumen: Este estudio pretende analizar la aplicación práctica del flipped classroom o clase invertida en varios grupos de 50 curso de Educación Primaria con la finalidad de comprobar qué diferencias existen entre la implementación de un modelo de enseñanzatradicional y un model o de enseñanza activo en el aula. Paraello, se realizó un grupo de discusión entrelosmaestros con el objetivo de recabar información sobre las características de los alumnos y del funcionamiento de cada grupo. Esto permitió implementar esta metodología a dos de los tres grupos participantes, siendo uno de ellos considerado el grupo control. El diseño de la unidad didáctica requirió la creación de un por tal web con información y vídeos sobre los contenidos abordados, en este caso, relacionados con la prácticadel balonmano. Los resultados de laevaluación muestran un rendimiento mayor en los grupos en los que se ha implementado el flipped dlassroom en comparación con el grupo que no lo aplica. Además, la alta participación en la clase inversa favorece el desarrollo de nuevas habilidades motrices y una mejora de la competencia social. De estos resultados se extraen como conclusiones que la aplicación del flipped classroom favorece el afianzamiento de los lazos entre profesor-alumno, así como un acercamiento de las familias hacia el estudio de sus hijos, fomentando la cooperación y colaboración entre familias y el centro educativo. Asimismo, ha mejorado la competencia motriz y social, además de la implicación y la motivación del alumnado, convirtiendo al estudiante como agente activo de su aprendizaje.
\end{abstract}

Palabras clave: Investigación educativa, educación física, métodos de enseñanza, tecnología educativa, desarrollo motor, motivación.

\begin{abstract}
This study aims to analyze the practical application of the flipped classroom or reverse class in various groups of the 5th year of Primary Education in order to check what differences exist between the implementation of a traditional teaching model and an active teaching model in the classroom. For this, a discussion group was held among the teachers in order to collect information on the characteristics of the students and the operation of each group. This allowed two of the three participating groups to implement this methodology, one of them being considered the control group. The design of the teaching unit required the creation of a web portal with information and videos on the content addressed, in this case, related to the practice of handball. The evaluation results show a higher performance in the groups in which the flipped classroom has been implemented compared to the group that does not apply it. Furthermore, high participation in the reverse class favors the development of new motor skills and an improvement in social competence. From these results, it is concluded that the application of the flipped classroom favors the strengthening of the ties between teacher-student, as well as an approach of families towards the study of their children, promoting cooperation and collaboration between families and the educational center. Likewise, motor and social competence have improved, in addition to the involvement and motivation of the students, making the student an active agent of their learning.
\end{abstract}

Keywords: Educational research, physical education, teaching methods, educational technology, motor development, motivation.

\section{Introducción}

La O rganización M undial de la Salud (OMS) recomienda que los menores entre los 5 y 17 años deben dedicar al menos 60 minutos aproximadamente al día al desempeño de actividades físicas de intensidad modera da a alta, así como evitar actividades sedentarias (OM S, 2020). Con la finalidad de atender a la salud y procurar el máximo desarrollo personal y social de la población

\footnotetext{
Fecha recepción: 01-09-20. Fecha de aceptación: 18-03-21 Nerea Felgueras Custodio nerea.felgueras@urjc.es
}

infantojuvenil, resulta de gran importancia resaltar la labor de la educación para con este objetivo y más concretamente, el papel queejercelaEducación Física (Real Decreto 124, 2014).

En la actualidad existe una tendencia hacia la incorporación de la tecnología en la educación, lo que deriva en la reflexión sobre los modelos educativos y la aparición de diversas metodologías educativas (MarquésM olías et al, 2019). De manera análoga, en el ámbito de la educación física se advierte una predisposición hacia la mejora de las intervenciones educativas mediante la implementación de alternativas pedagógicas 
innovadoras, otorgando al estudiante un papel más activo en el proceso de enseñanza y aprendizaje (Lleixà, 2017; Santos y Serpa, 2020). De este modo, el entorno de aprendizaje y la motivación se convierten en dos condicionantes que, en el contexto educativo, determinan el proceso de enseñanza y aprendizaje y, al mismo tiempo, dependen de las estrategias metodológicas que se apliquen en el centro educativo (Cuenca Ruano et al., 2021; 0 sterlie y M ehus, 2020; Gómez-Carrasco et al., 2019; Prieto Martín, 2017). El flipped classroom 0 clase invertida se considera un método de enseñanza activo que persigue la inmersión explícita del discente en su proceso de aprendizaje, donde se combina la enseñanza tradicional con los avances en tecnología (Santosy Serpa, 2020; A dams Becker et al. , 2017). Además, permite destinar el tiempo del aula a la consolidación de conocimientos y habilidades previa revisión del contenido teórico. En este sentido, cuando la metodología está bien diseñada y se aplica correctamente se puede producir una sinergia entre lo mejor de ambos mundos: la educación tradicional y la implementación de la tecnología en el ámbito educativo (Karabatak y Polat, 2019; 0 sterliey M ehus, 2020; Zainuddin y Perera, 2017; Zheng et al., 2020). Asimismo, tomando en consideración que el flipped classroom presenta una elevada capacidad de adaptación a las demandas de la situación, es preciso explorar su aplicación en el área de la Educación Física (Sargen y Casey, 2019). A esta premisa se unen las escasas horas lectivas asignadas a la asignatura de Educación Física en el currículo de Educación Primaria, lo que hace que el abordaje en esta temática sea aún más pertinente.

Por su parte, considerando el Decreto 89 (2014), la Educación Física se presta de juegos y depor tes que sirven a los diferentes propósitos educativos a lo largo de toda la etapa de Educación Primaria. Asimismo, se promueve la actividad física y los deportes tanto individuales como colectivos por los beneficios que reportan: fomento de la disciplina, potencia la fuerza de voluntad y el espíritu de superación y promueve la colaboración y el respeto hacia las normas y reglas del juego (Decreto 89,2014$)$. De manera específica, para los cursos de cuarto, quinto y sexto de Educación Primaria se promueve la iniciación a la competición en deportes de equipo comúnmente conocidos, entre los que se encuentra el bal onmano. En general, este deporte contribuye a generar hábitos de práctica deportiva, además de favorecer el desarrollo motriz y social de los estudiantes (Robles Rodríguez y Robles Rodríguez, 2021). Con frecuencia, los docentes de Educación Física ad- vierten falta de interés hacia la práctica deportiva, por lo que es preciso adaptar las estrategias de enseñanza de forma que se vea incrementada la motivación y, en consecuencia, la tasa de participación (Cuenca Ruano et al., 2021; Robles Rodríguezy Robles Rodríguez, 2021).

En esta investigación, con el fin de descifrar las principales contribuciones que aporta el flipped classroom como estrategia metodológica, se realizarán compara tivas atendiendo a diferentes aspectos relativos al desa rrollo físico y social asociados al balonmano, como ma teria curricular de la asignatura de Educación Física en Educación Primaria. Este estudio se desarrollará en tres clases de 50 curso de Educación Primaria perteneciente a un Colegio de Educación Infantil y Primaria, en adelante CEIP, en la zona sur de la Comunidad de Madrid. En dos de estas clases se enseñará el deporte del balonmano aplicando la estrategia metodológica flipped classroom y en la otra, primará la enseñanza tradicional.

El objetivo vertebrador de esta investigación reside en determinar cómo afecta la aplicación del modelo pedagógico flipped classroom en el proceso de enseñanza aprendizaje de habilidades físicas y sociales y aspectos normativos relativos al balonmano en alumnos de Educación Primaria en el área de Educación Física. Por su parte, como hipótesis de estudio se considera que la implementación del flipped classroom supone una mejora en la adquisición de contenidos, además de un aumento en la participación de los estudiantes, aspecto que correlaciona de manera positiva con la adquisición de aprendizajes y aumento de la motivación.

\section{Metodología}

El enfoque metodológico de la investigación se centra en la metodología cuantitativa, siguiendo un diseño experimental, pues la asignación de la muestra no responde a las leyes del azar. Es decir, la decisión de implementación de la metodología flipped classroom reside en los investigadores. El estudio pretende abordar cómo afecta la implementación del flipped classroom en el rendimiento académico de los estudiantes mediante el registro sistemático de conductas a través de una fichaderecogidadeinformación (Solano Fernández, 2017). Este instrumento de recogida de información fue sometido a la valoración de juicio de expertos para asegurar su validez de contenido. Para comprobar si existen cambios en las variables estudiadas, la recogida de información tendrá lugar antes del inicio de las sesiones basadas en el flipped classroom y una vez final izada la unidad didáctica del balonmano. 


\section{Procedimiento de muestreo y participantes}

La selección de la muestra se basa en un muestreo no probabilístico por conveniencia de los investigadores que atiende a la accesibilidad y a la disponibilidad de los participantes para colaborar en el estudio. Los participantes fueron los estudiantes de tres clases de quinto curso de la etapa de Educación Primaria de un CEIP de la zona sur de Madrid ( $n=65)$. La elección de la etapa educativa se debe a la experiencia previa de los estudiantes con al gunos de los contenidos curriculares abordados en la asignatura, por lo que ya estaban familiarizados con los deportes de equipo.

Cada uno de los grupos se ha designado con las letras $A, B$ y $C$, respectivamente. El flipped classroom se introdujo en los grupos $A(n=23)$ y $B(n=23)$, manteniéndose el grupo $C(n=19)$ con un método de enseñanza regular. Dicha diferenciación fue el resultado de una investigación curricular y actitudinal previa sobre la progresión académica de los alumnos, tanto en Educa ción Física, como en el resto de las asignaturas. Para este fin, tuvieron lugar varias reuniones con los profesores de 50 de Educación Primaria como vía de indagación y recolección de datos académicos y actitudinales de los estudiantes. Gracias a este trabajo previo se pudo concluir que los grupos A y C mostraban una progresión similar en el aprovechamiento y asimilación de los contenidos de las diferentes materias, así como una predisposición similar en la participación en las actividades propuestas. Por tanto, con el fin de que la investigación fuera lo más veraz y rigurosa posible, debía realizarse la comparativa metodológica entre estas dos clases, por presentar homogeneidad en sus características. Pues únicamente si el grupo control mantiene las características substanciales con el grupo experimental, se puede presuponer que la manipulación de la variable independiente tiene un efecto en la dependiente. Por tanto, se introdujo el flipped classroom en el grupo A, siendo el grupo experimental, y se mantuvo la clase regular u ordinaria en el grupo $\mathrm{C}$, tomándose como grupo control. De este modo, se posibilita comprobar si las diferencias en el rendimiento académico y en el desempeño actitudinal entre el grupo $\mathrm{A}$ y $\mathrm{C}$ se deben a la introducción del flipped classroom.

De manera paralela a la investigación principal, se realizó otro estudio con el grupo $B$. Esto se debe a que este grupo mostraba una progresión académica menor en comparación con los grupos A y C, por lo que se determinó introducir esta estrategia metodológica para observar su respuesta a la misma como un posible factor de mejora de la calidad educativa.

\section{Variables}

Para el establecimiento de las variables a valorar dentro de la propuesta, se estructuró el desarrollo motor del balonmano en sus cinco elementos más básicos, manejo, bote, Ianzamiento, recepción y defensa. Por otro lado, los aspectos del desarrollo normativo y social se organizan con respecto al cumplimiento de reglas y participación.

Se describen brevemente cada una de las variables seleccionadas en este estudio en laTabla 1.

\begin{tabular}{|c|c|c|}
\hline $\begin{array}{l}\text { Variable } \\
\text { dependiente }\end{array}$ & $\begin{array}{l}\text { Items de } \\
\text { evaluación }\end{array}$ & Descripción del constructo \\
\hline \multirow[t]{5}{*}{ Desar rollo motor } & Manejo & $\begin{array}{l}\text { Capacidad del alumno para dominar el balón sin que se le } \\
\text { escape de las manos. }\end{array}$ \\
\hline & Bote & $\begin{array}{l}\text { Capacidad de avanzar con el balón de un lugar a otro, } \\
\text { poniéndolo en contacto con el suelo, cuando el pase es } \\
\text { peligroso o imposible. }\end{array}$ \\
\hline & Lanzamiento & $\begin{array}{l}\text { Capacidad del alumno de superar al portero con el balón } \\
\text { introduciéndolo dentro de la portería. }\end{array}$ \\
\hline & Recepción & $\begin{array}{l}\text { Coordinación necesaria para bloquear el balón con una o dos } \\
\text { manos. }\end{array}$ \\
\hline & Defensa & Capacidad de contener y rechazar el ataque del equipo rival. \\
\hline \multirow[t]{3}{*}{$\begin{array}{l}\text { Desar rollo social } \\
\text { y normativo }\end{array}$} & Respeto & $\begin{array}{l}\text { Actitud respetuosa y empática con cada uno de los } \\
\text { compañeros/as. }\end{array}$ \\
\hline & Reglas & $\begin{array}{l}\text { Conocimiento y demostración durante la práctica de las } \\
\text { normas y reglas del balonmano. }\end{array}$ \\
\hline & Participación & Intervención activa durante la práctica del balonmano. \\
\hline
\end{tabular}

\section{Instrumentos}

La recogida de datos se realizó mediante observa ción directa de la ejecución de conductas a través de una ficha de recogida de información sometida a juicio de expertos a través del método Delphi. Para ello, en primer lugar, se presentó a los siete expertos participantes las categorías de análisis, basadas en la bibliografía revisada. Fueron necesarias 3 rondas de consulta al panel de expertos para alcanzar el consenso. En cada una de las rondas se implementaron las modificaciones pertinentes propuestas por los miembros del panel. La decisión de finalización del proceso iterativo entre rondas consecutivas atiende, por un lado, al criterio de consenso y, por otro, al criterio de estabilidad. El estadístico aplicado para decidir el consenso fue el rango intercuartílico relativo (RIR) y para determinar la estabilidad se aplicó la variación del rango intercuartílico relativo. En la tercera ronda de consulta el RIR y la variación del RIR cumplía con los criterios para dar por

Tabla 2

Items de la ficha de recogida de información İtems Indicadores de evaluación
$0=$ no $\quad 1=$ en $\quad 2=$ en $\quad 3=$ conseguido proceso proceso sin conseguido
con ayuda apoyo

Desplazamiento botando con el balón

Progreso en el manejo del balón.

Precisión en el lanzamiento del balón.

Recepción del balón en parado y en movimiento.

Capacidad de evitar o dificultar la progresión de

contrario en situaciones de juego.

Respeto a las reglas del juego y aceptación del resultado.

Valora su esfuerzo personal y el de los demás

Participación activa en los diferentes juegos

propuestos. 
finalizado el proceso iterativo. Por su parte, las varia bles que final mente se incluyeron en la ficha obtuvieron un coeficiente de variación menor o igual al $20 \%$ entre las decisiones de los expertos participantes. Una vez aplicado el método Delphi, la ficha de recogida de información se estructura en ocho ítems que responden a unos indicadores de evaluación. Estos aparecen en la Tabla 2.

\section{Procedimiento}

En primer lugar, se diseñó una unidad didáctica, en adelante U. D., sobre el deporte del balonmano, compuesta por 8 sesiones. A continuación, se establecieron y organizaron las reglas básicas del balonmano que debían conocer los alumnos para poder iniciar la práctica de este deporte y seguir una progresión a lo largo de las sesiones. De manera paralela, se creó el contenido audiovisual, incorporando animaciones junto con la intervención del profesor en la explicación de las normas básicas del balonmano. El vídeo se trasladó a los alumnos a través de un blog, que podrían acceder a él a tra vés de Internet, previo consentimiento informado firmado de la madre, padre o tutor/ a legal. Asimismo, el contenido incorporado en el blog incluía las actividades a desarrollar en las sesiones y vídeos adicionales sobre la práctica del balonmano. De este modo, se facilita la comprensión previa de los contenidos a abordar en la próxima sesión.

De manera simultánea, se diseñó la ficha de recogida de evaluación aplicando el método Delphi mencionado anteriormente. Una vez que todos los recursos estuvieron disponibles, se llevó a cabo la enseñanza siguiendo una metodología basada en el flipped classroom. La evaluación fue llevada a cabo por dos observadores externos diferentesalosinvestigadores del estudio, cuyos perfiles profesionales eran maestro de Educación Primaria especialista en Educación Física y psicólogo deportivo. Por su parte, la recogida de información tuvo lugar durante la primera y última sesión planificada de laU. D.

\section{Análisis estadístico de datos}

La técnica de análisis de datos se ha centrado, por un lado, en la exposición de estadísticos descriptivos de tendencia central (media, moda y mediana) y de dispersión (desviación típica, rango) a través de SPSSV 27. Por otro lado, puesto que no se puede considerar la distribución normal de la muestra, ni el criterio de homogeneidad y que la muestra de cada grupo es pequeña ( $n$ $<30$ ), se han aplicado tres pruebas no paramétricas. En primer lugar, se aplica la prueba $\mathrm{H}$ de Kruskal-Wallis para varias muestras independientes, con la finalidad de conocer si existen diferencias significativas en el rendimiento entre los tres grupos A, B y C. Por otro lado, la prueba de suma de rangos deW ilcoxon para dos muestras relacionadas permitirá conocer si ha habido una mejora significativa entre los momentos de evaluación pre/ post en cada uno de los grupos ( $A, B$ y $C$ ). Por otro lado, para conocer si existen diferencias en el rendimiento general del grupo atendiendo a la metodología aplicada (flipped classroom/ tradicional) se aplica la prueba $U$ de Mann-W hitney para dos muestras independientes en los gruposA (grupo experimental) y C (grupo control). Asimismo, se aplica esta misma prueba para conocer si existen diferencias en el rendimiento de cada variable dependiendo de la metodología aplicada (flipped classroom/ tradicional), pero esta vez tomando en consideración los dos grupos con aplicación del flipped classroom $(A+B$, denominado grupo $F)$ y el grupo $C$ con aplica ción de docencia tradicional. Además, para profundizar en el análisis y poder conocer si existe variabilidad en el rendimiento académico de cada grupo (A y C) se aplica la prueba de reacciones extremas de Moses para dos muestras independientes.

\section{Resultados}

Los estadísticos descriptivos relativos a los tres grupos ( $A, B$ y $C$ ) en ambas evaluaciones se muestran a continuación. En la totalidad de las variables de los grupos con flipped classroom (A y B) una media superior en la evaluación post. Sin embargo, en el grupo C con docencia regular se obtuvo medias más bajas en comparación con los grupos A y B, además de obtener en la variable lanzamiento de la evaluación post una media inferior una vez finalizada la unidad didáctica. En las tablasTabla 3, Tabla 4 yTabla 5 se muestran estos resultados detalladamente.

Tomando en consideración que los estadísticos descriptivos no aportan información suficiente sobre el nivel de significatividad de los cambios entre ambas eva luaciones, así como entre la metodología de la docencia, se presentan a continuación los resultados obtenidos tras aplicar la prueba de $\mathrm{H}$ de Kruskal-Wallis para tres muestras independientes, la pruebaW ilcoxon (Z) para muestras relacionadas, y, por último, las pruebas $U$ de M ann-W hitney y de reacciones extremas de M oses para dos muestras independientes.

Teniendo en cuenta la idiosincrasia de las condiciones reales del estudio, se aplica la prueba $\mathrm{H}$ de K ruskal- 
Tabla 3

\begin{tabular}{lccccc}
\multicolumn{7}{l}{ Estadíticos descriptivos Pre Post del grupoA (fli pped classroom) } & & & \\
\hline Variables & Media & Moda & Mediana & SD & Rango \\
\hline Pre_manejo & 2.48 & 2 & 2 & .846 & 3 \\
Post_manejo & 3.09 & 3 & 3 & .793 & 2 \\
Pre_bote & 2.57 & 2 & 3 & .728 & 3 \\
Post_bote & 3.04 & 3 & 3 & .638 & 2 \\
Pre_lanzamiento & 2.78 & 3 & 3 & .795 & 3 \\
Post_lanzamiento & 3.26 & 3 & 3 & .541 & 2 \\
Pre_recepción & 2.78 & 3 & 3 & .736 & 3 \\
Post_recepción & 3.04 & 4 & 3 & .562 & 2 \\
Pre_defensa & 2.83 & 3 & 3 & .778 & 3 \\
Post_defensa & 2.96 & 3 & 3 & .767 & 2 \\
Pre_respeto & 2.91 & 3 & 3 & .996 & 3 \\
Post_respeto & 3 & 3 & 3 & .905 & 3 \\
Pre_reglas & 2.57 & 2 & 3 & .843 & 3 \\
Post_reglas & 3.22 & 3 & 3 & .736 & 2 \\
Pre_participación & 2.7 & 4 & 3 & 1.105 & 3 \\
Post_participación & 3.26 & 3 & 3 & .541 & 2 \\
\hline Not_Nation & & 3 & & &
\end{tabular}

Nota: $N$ válidos $=23 ; \mathrm{N}$ perdidos $=0$.

\begin{tabular}{|c|c|c|c|c|c|}
\hline Variables & Media & Moda & M ediana & SD & Rango \\
\hline Pre_manejo & 2.26 & 3 & 2 & .752 & 2 \\
\hline Post_manejo & 2.96 & 3 & 3 & .706 & 2 \\
\hline Pre bote & 2.43 & 3 & 3 & .662 & 2 \\
\hline Post_bote & 2.91 & 3 & 3 & .596 & 2 \\
\hline Pre_lanzamiento & 2.39 & 2 & 2 & .783 & 3 \\
\hline Post_lanzamiento & 2.96 & 2 & 3 & .825 & 2 \\
\hline Pre_recepción & 2.43 & 3 & 3 & .662 & 2 \\
\hline Post_recepción & 2.96 & 3 & 3 & .562 & 2 \\
\hline Pre_defensa & 2.3 & 2 & 2 & 635 & 3 \\
\hline Post̄ defensa & 2.91 & 3 & 3 & .733 & 2 \\
\hline Pre_respeto & 2.7 & 3 & 3 & .974 & 3 \\
\hline Post_respeto & 3.09 & 3 & 3 & .793 & 3 \\
\hline Pre_reglas & 2.09 & 2 & 2 & .596 & 2 \\
\hline Post reglas & 2.96 & 3 & 3 & .825 & 3 \\
\hline Pre_participación & 2.83 & 3 & 3 & .984 & 3 \\
\hline Post_participación & 3.43 & 4 & 4 & .728 & 2 \\
\hline \multicolumn{6}{|c|}{ Nota: $N$ válidos $=23 ; N$ perdidos $=0$} \\
\hline \multicolumn{6}{|c|}{$\begin{array}{l}\text { Tabla } 5 \\
\text { Estadísticos descriptivos Pre-Post del grupo C (docencia tradicional) }\end{array}$} \\
\hline Variables & Media & Moda & Mediana & SD & Rango \\
\hline Pre manejo & 2.11 & 2 & 2 & .658 & 2 \\
\hline Post manejo & 2.47 & 3 & 3 & .612 & 2 \\
\hline Pre_bote & 2.32 & 2 & 2 & .582 & 2 \\
\hline Posț bote & 2.42 & 2 & 2 & .507 & 1 \\
\hline Pre Ianzamiento & 2.53 & 3 & 3 & .513 & 1 \\
\hline Post_lanzamiento & 2.37 & 2 & 2 & .496 & 1 \\
\hline Pre_recepción & 2.21 & 2 & 2 & .419 & 1 \\
\hline Post_recepción & 2.37 & 2 & 2 & .496 & 1 \\
\hline Pre_defensa & 2 & 2 & 2 & .417 & 2 \\
\hline Post_defensa & 2.26 & 2 & 2 & .452 & 1 \\
\hline Pre respeto & 2.95 & 3 & 3 & .780 & 3 \\
\hline Post respeto & 3 & 3 & 3 & .745 & 2 \\
\hline Pre_reglas & 1.58 & 2 & 2 & .507 & 1 \\
\hline Post reglas & 2.21 & 2 & 2 & .419 & 1 \\
\hline Pre participación & 2.79 & 2 & 3 & .976 & 3 \\
\hline Post participación & 2.84 & 3 & 3 & .898 & 3 \\
\hline
\end{tabular}

Wallis para conocer si el promedio poblacional de cada muestra ( $A, B$ y $C$ ) es similar entre grupos o difiere entre ellos.

En laTabla 6, se presenta el estadístico H de KruskalWallis y su nivel crítico bilateral (Sig. Asintót. bilateral). Atendiendo a los datos presentados, en la mayoría de las variables se rechaza la hipótesis nula, lo que implica que existen diferencias significativas entre grupos (A, B y $C)$ en todas las variables excepto en respeto $(p=.88)$ y participación $(p=.53)$.

Tabla 6

Estadístico $H$ de Kruskall-Wall lis: análisis rendimiento entre grupos ( $A, B$ y $C$ )

Variable Estadístico $\mathrm{H}$ de Kruskal-Wallis Sig. Asintót. bilateral Decisión sobre $\mathrm{H}_{0}$

Manejo

Mane

Lanzamiento $\quad 16.162$

Recepción $\quad 15.245$

Defensa

Respeto

Reglas

Participación

11.477
.243

.243

5.873

$\begin{array}{ll}.032 & \text { Se rechaza } \mathrm{H}_{0} \\ .004 & \text { Se rechaza } \mathrm{H}_{0} \\ .000 & \text { Se rechaza } \mathrm{H}_{0} \\ .000 & \text { Se rechaza } \mathrm{H}_{0} \\ .003 & \text { Se rechaza } \mathrm{H}_{0} \\ .886 & \text { Se conserva } \mathrm{H}_{0} \\ .000 & \text { Se rechaza } \mathrm{H}_{0} \\ .053 & \text { Se conserva } \mathrm{H}_{0}\end{array}$

Nota. $n_{A}=23 ; n_{B}=23 ; n_{C}=19$. Si Sig. Asintót. bilateral $=.05$, entones se rechaza la hipótesis

nula $\left(H_{0}\right)$, es decir, existe variabil idad en el rendimiento de cada variable entre los grupos $A, B$ y
En las tablasTabla 7, Tabla 8 y Tabla 9, se muestra el estadístico deW ilcoxon y su nivel crítico bilateral (Sig. Asintót. bilateral) para las evaluaciones pre/ post del grupo A y B (flipped classroom) y grupo C (clase regular). Asimismo, se considera un nivel de confianza del $95 \%$ ( $p$ d» .05). Para la prueba deW ilcoxon, la hipótesis nula presupone que ambas muestras proceden de poblaciones que presentan la misma distribución de probabilidad o lo que es lo mismo, la misma mediana. Por ello, si la hipótesis nula se rechaza, se asume que existen diferencias significativas entre ambas evaluaciones.

El grupo A (flipped classoom) obtiene diferencias significativas en la evaluación post en comparación con la evaluación pre en todas las variables excepto en las va riables defensa $(p=.58)$ y respeto $(p=.48)$.

El grupo B (flipped classoom) advierte una diferencia significativa en todas las variables tras trabajar los contenidos de la unidad didáctica del balonmano, pues el nivel de significatividad es menor a .05. Por tanto, se acepta la hipótesis alternativa en la que se determina que las puntuaciones de ambas evaluaciones difieren significativamente. Si, además, se atiende a los valores de la diferencia positiva, se detecta que las puntuaciones de la evaluación post son mayores que las obtenidas en la evaluación pre.

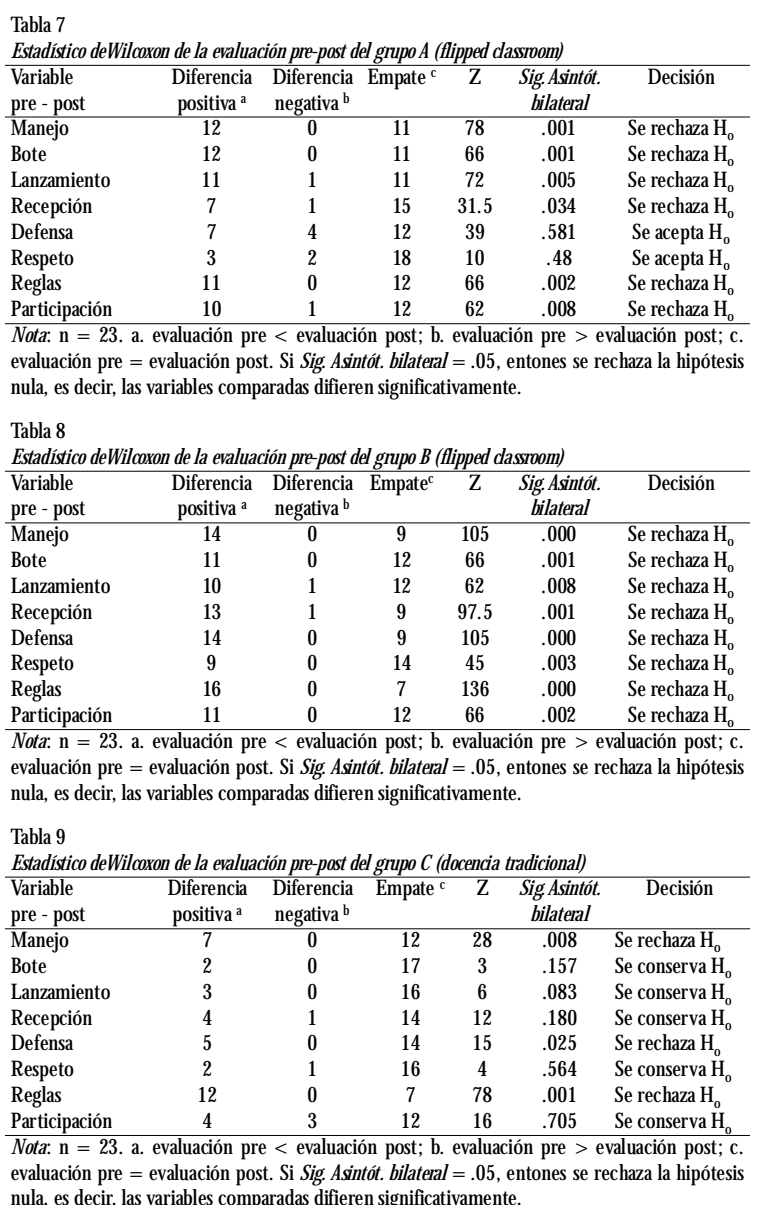


Por su parte, el grupo C, donde se imparte la docencia tradicional, no muestra diferencias significativas entre ambas eval uaciones en las variables bote $(p=.15)$, lanzamiento $(p=.083)$, recepción $(p=.18)$, respeto ( $p$ $=.564$ ) y participación ( $p=.705)$, siendo el nivel de significación de estas variables mayor 0 igual a .05. No obstante, en la evaluación post de las variables manejo $(p=.008)$, defensa $(p=.025)$ y reglas $(p=.001)$ se obtienen diferencias significativas en comparación con la evaluación pre, siendo el nivel de significatividad de estas tres variables superior a 05 .

Por otro lado, para conocer si existen diferencias generales en el rendimiento dependiendo de la metodología aplicada entre el grupo experimental A (flipped classroom) y el grupo control C (docencia tradicional) se aplica la prueba $U$ de Mann-W hitney para dos muestras independientes. Asimismo, para conocer si existe va riabilidad en el rendimiento dentro de cada uno de los grupos estudiados se aplica la prueba de reacciones extremas de M oses. El estadístico de contrasteU de M annW hitney, así como el estadístico de contraste de Moses se muestran en las tablas 10 y 11 con un nivel de confianza en ambas pruebas del $95 \%(p=.05)$.

En laTabla 10, se presenta el estadístico U de M annWhitney y su nivel crítico bilateral (Sig. Asintót. bilate ral). Se rechaza la hipótesis nula en la mayoría de las variables, lo que implica que existen diferencias significativas entre el grupo experimental ( $\mathrm{A}$ / flipped classroom) y el grupo control ( $C$ / tradicional) en el rendimiento de todas las variables excepto en las variables respeto ( $p$ $=.80)$ y participación $(p=.10)$. En la tabla 11 , se pre-

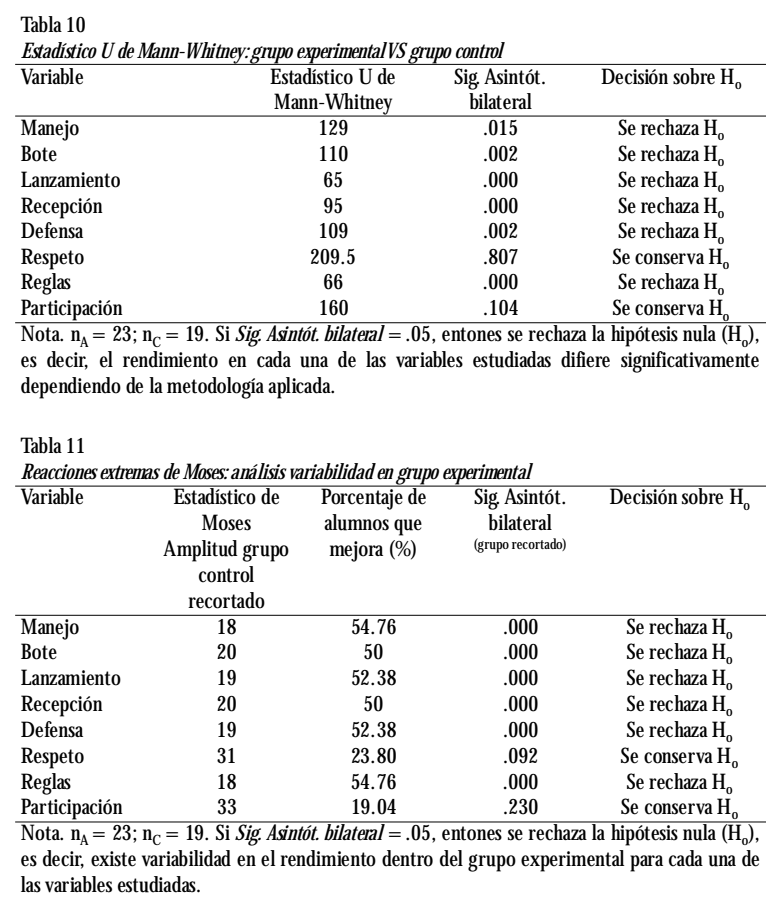

senta el estadístico reacciones extremas de Moses y su nivel crítico bilateral (Sig. Asintót, bilateral). Se observa que existen diferencias extremas en todas las variables estudiadas de los grupos en los que se aplica flipped classroom ( $A$ y $C$ ), excepto en las variables respeto ( $p=$ .09) y participación $(p=.23)$.

Finalmente, para conocer si existen diferencias significativas en el rendimiento de cada variable dependiendo de la metodología aplicada (flipped classroom/ tra dicional), de nuevo se aplica la prueba $U$ de MannW hitney para dos muestras independientes; pero esta vez considerando todos los casos de lametodologíaflipped classroom como unaúnica muestra $\left(n_{F}=46 ; n_{A}=23+n_{B}\right.$ $=23)$ y los casos de la metodología tradicional $\left(n_{c}=\right.$ 19). En la tabla 12, se observa que existen diferencias significativas en el rendimiento de cada variable estudiada según la metodología apl icada excepto en la varia ble defensa $(p=.66)$.

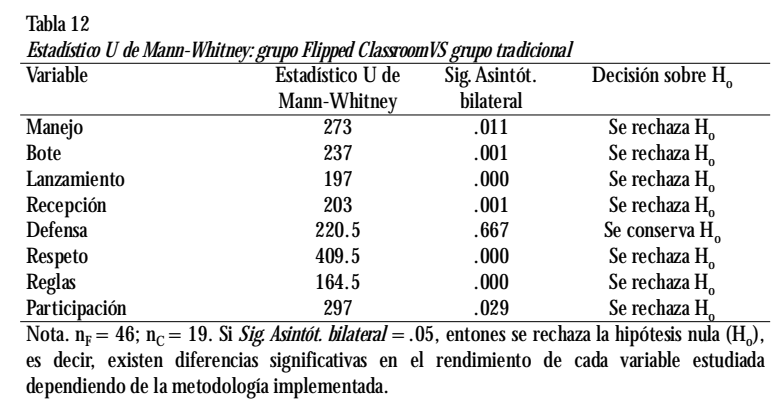

\section{Discusión}

En general, la experiencia de este estudio ha demostrado una mejora significativa en los resultados de aprendizaje de aquellos grupos en los que se ha implementado el flipped classroom entre los dos momentos de evaluación en la mayor parte de las variables. La aplicación de lasTIC a la asignatura de Educación Física puede favorecer el aprendizaje de conocimientos y conllevar a un aumento en la participación (0 sterlie y Mehus, 2020). Por su parte, en los grupos en los que se implementó esta estrategia metodológica se obtuvieron diferencias estadísticamente significativas en comparación con el grupo en el que se caracterizó por una docencia tradicional. Esta mejora podría presentar una casuística multifactorial. En primer lugar, la modifica ción del entorno de aprendizaje, incorporando elementos tecnológicos con los que el estudiante puede interactuar (p. ej. vídeos cuyo protagonista es el propio profesor que incorpora guiños a las tendencias propias de la edad escolar, posibilidad de interacción entre los contenidos del blog y el estudiante, etc. ) o dotando de flexibilidad al proceso de aprendizaje (p. ej. en tiempo, 
en número de visualizaciones, aplicando subtítulos, etc.) pueden contribuir a la mejora del rendimiento e incluso al aumento de la participación y de la motivación (Ramírez-M ontoyay Ramírez Hernández, 2016; Sagent y Casey, 2019; Portela, 2020). En segundo lugar, podría deberse a que los estudiantes tienen la capacidad de controlar la frecuencia de las visualizaciones del contenido teórico, así como el ritmo de visualización, otorgando a esta estrategia la capacidad de adaptarse a los diferentes ritmos de aprendizaje (A beysekera y Dawson, 2015; Sagent y Casey, 2019). 0 trosautores sostienen que cuanto mayor sea la implicación emocional en el proceso de aprendizaje más significativo será este y, por tanto, se producirá una generación de aprendizajes (Chi yWylie, 2014; W ittrock, 1992). También se ha observado en diferentes estudios que la aplicación del flipped classroom se asocia a un aumento en la motivación de los estudiantes, que se traduce en un incremento en su participación (Sagent y Casey, 2019; Steen-U theim y Foldnes, 2019; Hinojo et al., 2020; Portela, 2020). En contrapartida, otros autores mantienen que las dificultades en la autorregulación de los estudiantes durante el proceso de aprendizaje conllevan una disminución de la participación en las actividades propuestas (Awidi y Paynter, 2019). Por su parte, los estudiantes han podido obtener mejores resultados de aprendizaje puesto que se ha podido aprovechar el tiempo disponible en el aula para fomentar la participación y potenciar la interacción entre iguales, consolidando de este modo la práctica de las diferentes habilidades motrices y sociales trabajadas, así como de los contenidos teóricos (Alten et al., 2019; Gómez-García et al., 2019; Hinojo et al. 2020).

De manera específica, los resultados advierten que en el grupo en el que se implementa el flipped classroom existe una variabilidad intragrupo en el rendimiento de las habilidades y destrezas trabajadas, a pesar de que el progreso en la consolidación de aprendizajes es evidente a nivel general (Gómez-Carrasco et al., 2019).

Atendiendo al desarrollo de habilidades motrices (manejo, bote, lanzamiento, recepción y defensa), se observó una mejora significativa en los grupos en los que se implementó el flipped classroom en comparación con el grupo tradicional una vez finalizada la unidad didáctica. Por su parte, en cuanto al desarrollo social y normativo, se detecta una tendencia de mejora similar entre los dos tipos de metodología docente en las evaluaciones pre-post. En este sentido, es importante destacar que la variable participación muestra una mejora muy significativa para los grupos A y $B$, los dos grupos que aplican flipped classroom. De este modo, una posible explicación a la mejora de las habilidades motrices en los grupos A y B se deriva del aumento de la participa ción en el desarrollo de las sesiones donde se aplicó esta estrategia metodológica (Alten et al., 2019; GómezGarcía, 2019; Santos y Serpa, 2020; Sargent y Casey, 2019).

Una vez analizadas las mejoras obtenidas en el desarrollo de las capacidades motrices y sociales es posible predecir el desarrollo de una sinergia entre ambas, probablemente inducida por el elevado índice de participación de losestudiantes (Karabatak y Polay, 2019; Alten et al., 2019; Gómez-García, 2019).

\section{Conclusiones}

La introducción de esta nueva pedagogía supone un cambio innovador en la metodología de la enseñanza, así como en el proceso de enseñanza aprendizaje y en el rol que desempeñan docentes y discentes (Chiang et al., 2018). Además, desde un punto de vista más amplio, implica un cambio que afecta a toda la comunidad educativa.

Es necesario destacar, también, que la consiguiente estrategia metodológica, es aplicable a otros cursos y otras asignaturas, siempre que se realice con las correspondientes adaptaciones previas. En los estudios previos que se han descrito a lo largo de este trabajo, se observa que la aplicación práctica del flipped classroom obtiene mejoras en el rendimiento del alumnado de Educación Primaria, Secundaria, Bachillerato y de estudios universitarios, formando una parte esencial del proceso enseñanza-aprendizaje.

En esta investigación, se observa una mejora de las habilidades motrices y sociales en los grupos donde se ha implementado la clase inversa en comparación con los grupos que han recibido una educación tradicional. Además, se realizó una investigación paralela entre los dos grupos donde se implementa el flipped classroom, donde en una de ellas obtenía tanto en el área de Educa ción Física como en el resto de las asignaturas, calificaciones por debajo de la media de los grupos de 50 curso. Con este estudio se observa que estos dos grupos consiguen obtener un rendimiento muy similar, obteniendo una tendencia equivalente, tanto en el apartado motriz como en el social. Esto se debe a que en la participación, implicación y motivación del alumnado se obtiene unos resultados con una mejoría significativa. Asimismo, se puede llegar a la conclusión de que existe una sinergia entre las mejoras de las capacidades sociales y la mejora de las demás capacidades. 
Los alumnos, por otro lado, muestran una mayor autonomía, ya que el profesor se convierte en un guía del proceso enseñanza-aprendizaje. El alumnado se convierte en parte activa de su propio aprendizaje. Con esto se puede llegar a la conclusión de que el alumnado construye su propio camino hacia la excelencia.

Por último, el aumento de la participación derivada de la implementación del flipped classroom incita a los estudiantes a progresar tanto en la ejecución práctica como en el desarrollo teórico, trabajando de forma activa y en equipo en todas las sesiones. Esto tiene lugar de manera ascendente, es decir, se consigue que en las últimas sesiones se alcance un equilibrio y una relación entre iguales entre los compañeros el grupo. Esto se produce porque se crea un grupo con un ambiente de trabajo colaborativo.

\section{Referencias}

Abeysekera, L. \& Dawson, P. (2015). Motivation and cognitive load in the flipped classroom: definition, rationale and a call for research. Higher Education Research and Development, 34(1), 1-14. https:/ / doi.org/ 10.1080/ 07294360.2014.934336

Adams Becker, S., Cummins, M., Davis, A., Freeman, A., Hall Giesinger, C. \&A nanthanarayanan, V. (2017). N MC Horizon Report: 2017 Higher Education Edition. Austin, Texas: The New Media Consortium.

Alten, D. C. D., Phielix, C., Janssen, J. \& Kester, L. (2019). Effects of flipping the classroom on learning outcomesand satisfaction: ametaranalysis. Educational Research Review, 28, 1-18. https: / / doi.org/ 10.1016/ j.edurev.2019.05.003

Awidi, I.T. \& Paynter, M . (2019). The impact of aflipped classroom approach on student learning experience. Computers\& Education, 128, 269-283. https:/ / doi.org/ 10.1016/ j.compedu. 2018.09.013

Chi, M.T. H. \&W ylie, R. (2014). The ICAP framework: linking cognitive engagement to activate learning outcomes. Educational Psychologist, 49(4), 219-243. https:/ / doi.org/ 10.1080/ 00461520.2014.965823

Chiang, T. H. C., Yang, S. J. H., \&Yin, C. (2018). Effect of gender differences on 3-on-3 basketball games taught in a mobile flipped classroom. Interactive Learning Environments, 27(8), 1093-1105. https:/ / doi.org/ 10.1080/ 10494820.2018.1495652

Consejo de Gobierno (2014, 24 de julio). Decreto 89. Por el que se establece para la Comunidad de Madrid el Currículo de la Educación Primaria. Boletín O ficial de la Comunidad de Madrid 175. https:// www.bocm.es/ boletin/ CM_O rden_BOCM/ 2014/ 07/ 25/ BO CM-20140725-1.PDF

Cuenca Ruano, P., García M artínez, S., Ferriz Valero, A. \& Tortosa Martínez, J. (2021). Análisis compara tivo de los perfiles motivacionales y el Estado de Flow entre una metodología tradicional y la metodología Flipped Classroom en estudiantes de Educa ción Física. Retos, 39, 338-344. https:/ / doi.org/ 10.47197/ retos. v0i39. 78574

Gómez-Carrasco, C. J., M onteagudo-Fernández, J., Moreno-Vera, J. R. \& Sainz-Gómez, M. (2019). Effects of a Gamification and Flipped-Classroom Program for Teachers in Training on M otivation and Learning Perception. Education Sciences, 9 (299), 1-15. https: / / doi. org/ 10.3390/ educsci9040299

Gómez-García, J., Sellés, S. \& Ferriz-Valero, A. (2019). Flipped Classroom como propuesta en la mejora del rendimiento académico y motivación del alumnado en Educación Física. Kronos, 18(2), 1-12. https:/ / revistakronos.info/ articulos/ ?search-term $=\&$ volnum $=18-2$

Hinojo Lucena, F. J., López Belmonte, J., Fuentes Ca brera, A., Trujillo Torres, J. M. \& Pozo Sánchez, S. (2020). A cademic effects of the use of flipped learning in physical education. International Journal of Environmental Research and Public Health, 17(1), 276. https:/ / doi.org/ 10.3390/ ijerph17010276

Karabatak, S. \& Polat, H. (2019).Theeffects of the flipped classroom model designed according to the ARCS motivation strategies on the students' motivation and academic achievement levels. Education and InformationTechnologies, 25, 1475-1495. https:/ / doi.org/ 10.1007/ s10639-019-09985-1

Lleixá, T. (2017). Didáctica de la educación física: nuevostemas, nuevoscontextos. Didactae, (2), 2-5. https:/ / doi.org/ 10.1344/ did.2017.2.2-5

Marqués-M olías, L., Palau-Martín, R., U sart, M. \& Morilla, F. (2019). The Flipped classroom in the learning of korfball in fifth and sixth grade. Aloma, $37(2)$, 43-52. https:// doi.org/ 10.51698/ aloma. 2019.37.2.43-52

Ministerio de Educación, Cultura y Deporte (2014, 28 de febrero). Real Decreto 126. Por el que se establece el currículo básico de la Educación Primaria. Boletín O ficial del Estado 52. https:/ / www.boe.es/ diario_boe/ txt.php?id=BOE-A-2014-2222

O rganización M undial de laSalud (2020, 26 de noviembre). Actividad física. https: / / www.who.int/ es/ news-room/ fact-sheets/ detail/ physical-activity 0 sterlie, 0. \& M ehus, I. (2020). The Impact of Flipped 
Learning on Cognitive Knowledge Learning and Intrinsic M otivation in N orwegian Secondary Physical Education. Education Science, 10 (110), 1-16. https:/ / doi.org/ 10.3390/ educsci10040110

Portela, F. (2020). TechTeach-An innovate method to increase the students engagement at classrooms. Information, 11(10), 483. https:/ / doi.org/ 10.3390/ info11100483

Prieto Martín, A. (2017). Sección I. Fundamentación del modelo de aprendizaje inverso. Flipped learning. Aplicar el modelo de aprendizaje inverso, 21-90. Humanes, España: Editorial Narcea.

Ramírez-M ontoya, M. S. \& Ramírez Hernández, D. C. (2016). Inverted learning enviroments with technology, innovation and flexibility: students experience and meanings. Journal of Information Technology Research, 9(1), 18-33. https:/ / doi. org/ 10.4018/ JITR.2016010102

Robles Rodríguez, A. \& Robles Rodríguez, J. (2021). La participación en las clases de educación física la ESO y Bachillerato. Un estudio sobre un deporte tradicional (Balonmano) y un deporte alternativo (Tchoukball). Retos, 39, 78-83. https:/ / doi.org/ $10.47197 /$ retos. v0i39. 78129

Santos, A. I. \& Serpa, S. (2020). Flipped Classroom for and Active Learning. Journal of Education and e Learning Research, 7(2), 167-173. https:/ / doi. org/ 10.20448/ journal.509.2020.72.167.173

Santos, A. S. \& Serpa, S. (2020). Flipped classroom for active learning. Journal of Education and e-Learning Research, 7(2), 167-173. https:/ / doi.org/ 10.20448/ journal.509.2020.72.167.173

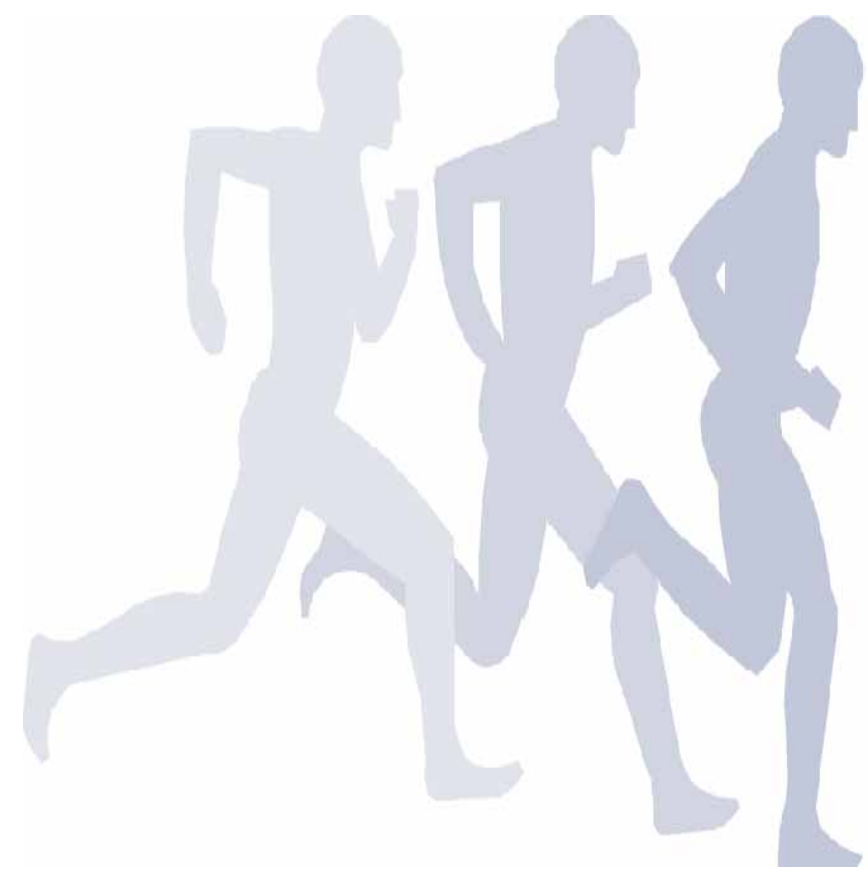

Sargent, J. \& Casey, A. (2019). Flipped learning, pedagogy and digital technology: establishing consistent practice to optimise lesson time. European Physical Education Review, 13. https:// doi.org/ 10.1177/ $1356336 \times 19826603$

Solano Fernández, I. (2017). Capítulo 5. Instrumentos de recogida de información. En Prendes Espinosa y González Calatayud (coords.). Trabajo Fin de Máster en Tecnología Educativa. O rientaciones para la elaboración y criterios de calidad. U niversidad de M urcia: Editum. https:/ / edit.um. es/ blog/ trabajo-fin-de-master-entecnologia-educativa-orientaciones-para-laelaboracion-y-criterios-de-calidad/

Steen-U theim, A. T. \& Foldnes, N. (2018). A qualitative investigation of student engagement in a flipped classroom. Teaching in Higher Education, 23(3), 307324. 13562517.2017.1379481

W ittrock, M. C. (1992). Generative learning processes of the brain. Educational Psychologist, 27(4), 531-541. https:/ / doi.org/ 10.1207/ s15326985ep2704_8

Zainuddin, Z. \& Perera, C. J. (2017). Exploring stüdents' competence, autonomy and relatednessin the flipped classroom pedagogical model. Journal of Further and Higher Education, 43(1), 115-126. https:/ / doi. org/ 10.1080/ 0309877X.2017.1356916

Zheng, L., Bhagat, K. K., Zhen, Y. \& Zhang, X. (2020). The Effectiveness of the Flipped Classroom on Students' Learning Achievement and Learning Motivation: A MetaAnalysis. Journal of Educational Technology \& Society, 23(1), 1-15. https:/ / www.jets. net/ collection/ published-issues/ 23_1

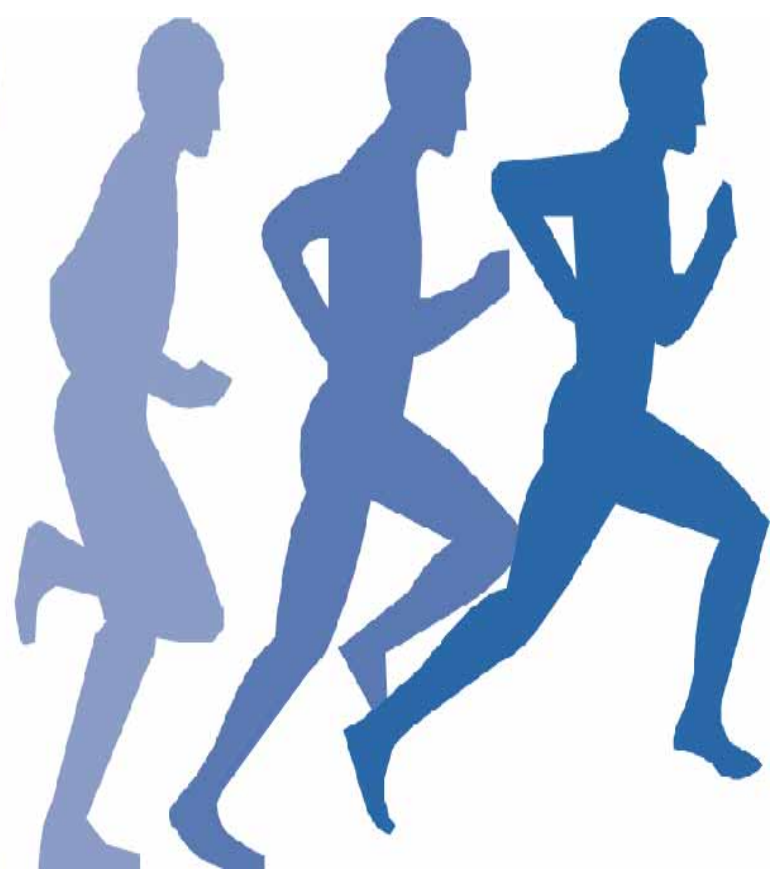

